\title{
Improved Correlation Coefficients of Quadripartitioned Neutrosophic Pythagorean sets for MADM
}

Radha $\mathrm{R}^{1}$ and Stanis Arul Mary $\mathrm{A}^{*, 2}$

Received: 27 February 2021/ Accepted: 12 May 2021/ Published online: 18 June 2021

(C)Sacred Heart Research Publications 2017

\begin{abstract}
Quadripartitioned single valued neutrosophic pythagorean sets is a improvisation of Wang's single valued neutrosophic sets. In this paper we have studied the improved correlation coefficients of quadripartitioned single valued neutrosophic pythagorean sets and investigate its properties. Further we have applied this concept in multiple attribute decision making methods with quadripartitioned single valued neutrosophic pythagorean environment. Finally we illustrated an example in the above proposed method to the multiple attribute decision making problems.
\end{abstract}

Key words: Quadripartitioned Neutrosophic Pythagorean Sets, Quadripartitioned Neutrosophic Sets, Improved Correlation Coefficient.

AMS classification: $60 \mathrm{~A} 86,62 \mathrm{~A} 86$

\section{Introduction}

Fuzzy sets were introduced by Zadeh [21] in 1965 which allows the membership function valued in the interval $[0,1]$ and also it is an extension of classical set theory. Fuzzy set helps to deal the concept of uncertainty, vagueness and imprecision which is not possible in the cantorian set. As an extension of Zadeh's fuzzy set theory intuitionistic fuzzy set(IFS) was introduced by Atanassov [1] in 1986, which consists of degree of membership and degree of non membership and lies in the interval of $[0,1]$. IFS theory widely used in the areas of logic programming, decision making problems, medical diagnosis etc.

Florentin Smarandache [13] introduced the concept of Neutrosophic set in 1995 which provides the knowledge of neutral thought by introducing the new factor

\footnotetext{
1,2 Department of Mathematics, Nirmala College for Women, Coimbatore, India.

Email: ${ }^{1}$ radharmat2020@gmail.com, ${ }^{*}, 2$ kathirsubbu1987@gmail.com
} 
called indeterminacy in the set. Therefore neutrosophic set was framed and it includes the components of truth membership function(T), indeterminacy membership function(I), and falsity membership function(F) respectively. Neutrosophic sets deals with non standard interval of $]^{-} 01^{+}$. Since neutrosophic set deals the indeterminacy effectively it plays an vital role in many applications areas include information technology, decision support system, relational database systems, medical diagnosis, multicriteria decision making problems etc.,

To deal the real world problems, Wang [12](2010) introduced the concept of single valued neutrosophic sets(SVNS) which is also known as an extension of intuitionistic fuzzy sets and it became a very new hot research topic now. Rajashi Chatterjee.,et al [12] proposed the concept of Quadripartitioned single valued neutrosophic sets which is based on Belnap's four valued logic and Smarandache's four numerical valued logic. In (QSVNS) indeterminacy is splitted into two functions known as 'Contradicition' (both true and false) and 'Unknown' (neither true nor false) so that QSVNS has four components T,C,U,F which also lies in the non standard unit interval $]^{-} 01^{+}$. Further, R. Radha and A. Stanis Arul Mary defined a new hybrid model of Quadripartitioned Neutrosophic Pythagorean Sets in 2021.

Correlation coefficient is a effective mathematical tool to measure the strength of the relationship between two variables. So many researchers pay the attention to the concept of various correlation coefficients of the different sets like fuzzy set, IFS, SVNS, QSVNS. In 1999 D.A Chiang and N.P.Lin [3] proposed the correlation of fuzzy sets under fuzzy environment. Later D.H.Hong [4](2006) defined fuzzy measures for a correlation coefficient of fuzzy numbers under Tw (the weakest t-norm) based fuzzy arithmetic operations. Correlation coefficients plays an important role in many real world problems like multiple attribute group decision making, clustering analysis, pattern recognition, medical diagnosis etc., Jun Ye[20 ] defined the improved correlation coefficients of single valued neutrosophic sets and interval neutrosophic sets for multiple attribute decision making to overcome the drawbacks of the correlation coefficients of single valued neutrosophic sets (SVNSs) which is defined in [16].

In this paper, We have discussed some of its properties and decision making method using the improved correlation coefficient with quadripartitioned neutrosophic pythagorean environment. Additionaly,an illustrative example is given in above proposed correlation method particularly in multiple criteria decision making problems. 
ISSN: 2456-8686, 5(1), 2021:142-153

https://doi.org/10.26524/cm101

\section{Preliminaries}

Definition 2.1 (13) Let X be a universe. A Neutrosophic set A on X can be defined as follows:

$$
A=\left\langle\left\{x, T_{A}(x), I_{A}(x), F_{A}(x)\right\rangle: x \in X\right\}
$$

Where $T_{A}, I_{A}, F_{A}: U \rightarrow[0,1]$ and $0 \leq T_{A}(x)+I_{A}(x)+F_{A}(x) \leq 3$

Here, $T_{A}(x)$ is the degree of membership, $I_{A}(x)$ is the degree of inderminancy and $F_{A}(x)$ is the degree of non-membership.

Here, $T_{A}(x)$ and $F_{A}(x)$ are dependent neutrosophic components and $I_{A}(x)$ is an independent component.

Definition 2.2 (10) Let $\mathrm{X}$ be a universe. A Quadripartitioned neutrosophic pythagorean set A with T, F, C and $\mathrm{U}$ as dependent neutrosophic components for A on $\mathrm{X}$ is an object of the form

$$
A=\left\{\left\langle x, T_{A}, C_{A}, U_{A}, F_{A}\right\rangle: x \in X\right\}
$$

Where $T_{A}+F_{A} \leq 1, C_{A}+U_{A} \leq 1$ and

$$
\left(T_{A}\right)^{2}+\left(C_{A}\right)^{2}+\left(U_{A}\right)^{2}+\left(F_{A}\right)^{2} \leq 2
$$

Here, $T_{A}(x)$ is the truth membership, $C_{A}(x)$ is contradiction membership, $U_{A}(x)$ is ignorance membership, $F_{A}(x)$ is the false membership

Definition 2.3 (12) Let P be a non-empty set.A Quadripartitioned neutrosophic set $\mathrm{A}$ over $\mathrm{P}$ characterizes each element $\mathrm{p}$ in $\mathrm{P}$ a truth -membership function $T_{A}$, a contradiction membership function $C_{A}$, ignorance membership function $U_{A}$ and a false membership function $F_{A}$, such that for each $\mathrm{p}$ in $\mathrm{P}$

$$
T_{A}+C_{A}+U_{A}+F_{A} \leq 4
$$

Definition 2.4 (10) The complement of a quadripartitioned neutrosophic pythagorean set $(\mathrm{F}, \mathrm{A})$ on $\mathrm{X}$ Denoted by $(F, A)^{c}$ and is defined as $F^{c}(x)=\left\{\left\langle x, F_{A}(x), U_{A}(x), C_{A}(x), T_{A}(x)\right\rangle: x \in X\right\}$.

Definition 2.5 (10) Let $A=\left\langle x, T_{A}(x), C_{A}(x), U_{A}(x), F_{A}(x)\right\rangle$ and 
ISSN: 2456-8686, 5(1), 2021:142-153

https://doi.org/10.26524/cm101

$B=\left\langle x, T_{B}(x), C_{B}(x), U_{B}(x), F_{B}(x)\right\rangle$ are quadripartitioned neutrosophic pythagorean sets. Then

$$
\begin{aligned}
A \cup B=\left\langle x, \max \left(T_{A}(x),\right.\right. & \left.T_{B}(x)\right), \max \left(C_{A}(x), C_{B}(x)\right), \\
& \left.\min \left(U_{A}(x), U_{B}(x)\right), \min \left(F_{A}(x), F_{B}(x)\right)\right\rangle \\
A \cap B=\left\langle x, \min \left(T_{A}(x),\right.\right. & \left.T_{B}(x)\right), \min \left(C_{A}(x), C_{B}(x)\right), \\
& \left.\max \left(U_{A}(x), U_{B}(x)\right), \max \left(F_{A}(x), F_{B}(x)\right)\right\rangle .
\end{aligned}
$$

\section{Improved Correlation Coefficients}

Definition 3.1 Let $\mathrm{P}$ and $\mathrm{Q}$ be any two QNPS s $\mathrm{s}$ in the universe of discourse $R=\left\{r_{1}, r_{2}, r_{3}, \cdots, r_{n}\right\}$, then the improved correlation coefficient between $\mathrm{P}$ and $\mathrm{Q}$ is defined as follows

$$
K(P, Q)=\frac{1}{4 n} \sum_{k=1}^{n}\left[\mu_{k}\left(1-\triangle T_{k}\right)+\varphi_{k}\left(1-\triangle C_{k}\right)+\gamma_{k}\left(1-\triangle U_{k}\right)+v_{k}\left(1-\triangle F_{k}\right)\right]
$$

Where

$$
\begin{gathered}
\mu_{k}=\frac{2-\triangle T_{k}-\triangle T_{\max }}{2-\triangle T_{\min }-\triangle T_{\max }}, \\
\varphi_{k}=\frac{2-\triangle C_{k}-\triangle C_{\text {max }}}{2-\triangle C_{\min }-\triangle C_{\max }}, \\
\gamma_{k}=\frac{2-\triangle U_{k}-\triangle U_{\max }}{2-\triangle U_{\min }-\triangle U_{\max }}, \\
v_{k}=\frac{2-\triangle F_{k}-\triangle F_{\max }}{2-\triangle F_{\min }-\triangle F_{\max }}, \\
\triangle T_{K}=\left|T_{P}^{2}\left(r_{k}\right)-T_{Q}^{2}\left(r_{k}\right)\right|, \\
\triangle C_{K}=\left|C_{P}^{2}\left(r_{k}\right)-C_{Q}^{2}\left(r_{k}\right)\right|, \\
\triangle U_{K}=\left|U_{P}^{2}\left(r_{k}\right)-U_{Q}^{2}\left(r_{k}\right)\right|, \\
\triangle F_{K}=\left|F_{P}^{2}\left(r_{k}\right)-F_{Q}^{2}\left(r_{k}\right)\right|, \\
\triangle T_{\min }=\min _{k}\left|T_{P}^{2}\left(r_{k}\right)-T_{Q}^{2}\left(r_{k}\right)\right|, \\
\triangle C_{\min }=\min _{k}\left|C_{P}^{2}\left(r_{k}\right)-C_{Q}^{2}\left(r_{k}\right)\right|, \\
\triangle U_{\min }=\min _{k}\left|U_{P}^{2}\left(r_{k}\right)-U_{Q}^{2}\left(r_{k}\right)\right|,
\end{gathered}
$$


ISSN: 2456-8686, 5(1), 2021:142-153

https://doi.org/10.26524/cm101

$$
\begin{aligned}
& \triangle F_{\text {min }}=\min _{k}\left|F_{P}^{2}\left(r_{k}\right)-F_{Q}^{2}\left(r_{k}\right)\right|, \\
& \triangle T_{\text {max }}=\max _{k}\left|T_{P}^{2}\left(r_{k}\right)-T_{Q}^{2}\left(r_{k}\right)\right|, \\
& \triangle C_{\max }=\max _{k}\left|C_{P}^{2}\left(r_{k}\right)-C_{Q}^{2}\left(r_{k}\right)\right|, \\
& \triangle U_{\text {max }}=\max _{k}\left|U_{P}^{2}\left(r_{k}\right)-U_{Q}^{2}\left(r_{k}\right)\right|, \\
& \triangle F_{\text {max }}=\max _{k}\left|F_{P}^{2}\left(r_{k}\right)-F_{Q}^{2}\left(r_{k}\right)\right|,
\end{aligned}
$$

For any $r_{k} \in R$ and $k=1,2,3, \cdots, n$.

Theorem 3.2 For any two QNPS s P and Q in the universe of discourse $R=\left\{r_{1}, r_{2}, r_{3}, \cdots, r_{n}\right\}$, the improved correlation coefficient $\mathrm{K}(\mathrm{P}, \mathrm{Q})$ satisfies the following properties.

(i) $\mathrm{K}(\mathrm{P}, \mathrm{Q})=\mathrm{K}(\mathrm{Q}, \mathrm{P})$;

(ii) $0 \leq K(P, Q) \leq 1$;

(iii) $\mathrm{K}(\mathrm{P}, \mathrm{Q})=1$ iff $\mathrm{P}=\mathrm{Q}$.

\section{Proof:}

(i) It is obvious and straightforward.

(ii) Here, $0 \leq \mu_{k} \leq 1, \quad 0 \leq \varphi_{k} \leq 1, \quad 0 \leq \gamma_{k} \leq 1, \quad 0 \leq v_{k} \leq 1$,

$0 \leq 1-\triangle T_{k} \leq 1, \quad 0 \leq 1-\triangle C_{k} \leq 1, \quad 0 \leq 1-\triangle U_{k} \leq 1, \quad 0 \leq 1-\triangle F_{k} \leq 1$,

Therefore the following inequation satisfies

$0 \leq \mu_{k}\left(1-\triangle T_{k}\right)+\varphi_{k}\left(1-\triangle C_{k}\right)+\gamma_{k}\left(1-\triangle U_{k}\right)+v_{k}\left(1-\triangle F_{k}\right) \leq 5$.

Hence we have $0 \leq K(P, Q) \leq 1$.

(iii) If $\mathrm{K}(\mathrm{P}, \mathrm{Q})=1$, then we get

$\mu_{k}\left(1-\triangle T_{k}\right)+\varphi_{k}\left(1-\triangle C_{k}\right)+\gamma_{k}\left(1-\triangle U_{k}\right)+v_{k}\left(1-\triangle F_{k}\right)=4$.

Since $0 \leq \mu_{k}\left(1-\triangle T_{k}\right) \leq 1,0 \leq \varphi_{k}\left(1-\triangle C_{k}\right) \leq 1,0 \leq \gamma_{k}\left(1-\triangle U_{k}\right) \leq 1$ and $0 \leq v_{k}\left(1-\triangle F_{k}\right) \leq 1$, there are $\mu_{k}\left(1-\triangle T_{k}\right)=1, \varphi_{k}\left(1-\triangle C_{k}\right)=1$,

$\gamma_{k}\left(1-\triangle U_{k}\right)=1$ and $v_{k}\left(1-\triangle F_{k}\right)=1$.

And also since $0 \leq \mu_{k} \leq 1,0 \varphi_{k} \leq 1,0 \leq \gamma_{k} \leq 1$ and $0 \leq v_{k} \leq 1$,

$0 \leq 1-\triangle T_{k} \leq 1,0 \leq 1-\triangle C_{k} \leq 1,0 \leq 1-\triangle U_{k} \leq 1,0 \leq 1-\triangle F_{k} \leq 1$.

We get $\mu_{k}=\varphi_{k}=\delta_{k}=\gamma_{k}=v_{k}=1$ and

$1-\triangle T_{k}=1-\triangle C_{k}=1-\triangle U_{k}=1-\triangle F_{k}=1$.

This implies, $\triangle T_{k}=\triangle T_{\min }=\triangle T_{\max }=0$,

$\triangle C_{k}=\triangle C_{\text {min }}=\triangle C_{\max }=0, \triangle U_{k}=\triangle U_{\min }=\triangle U_{\max }=0$, 
ISSN: 2456-8686, 5(1), 2021:142-153

https://doi.org/10.26524/cm101

$\triangle F_{k}=\triangle F_{\min }=\triangle F_{\max }=0$. Hence $T_{P}\left(r_{k}\right)=T_{Q}\left(r_{k}\right)$,

$C_{P}\left(r_{k}\right)=C_{Q}\left(r_{k}\right), U_{P}\left(r_{k}\right)=U_{Q}\left(r_{k}\right)$ and $F_{P}\left(r_{k}\right)=F_{Q}\left(r_{k}\right)$ for any $r_{k} \in R$ and $k=1,2,3 \cdots, n$.Hence $\mathrm{P}=\mathrm{Q}$.

Conversely, assume that $\mathrm{P}=\mathrm{Q}$, this implies $T_{P}\left(r_{k}\right)=T_{Q}\left(r_{k}\right)$,

$C_{P}\left(r_{k}\right)=C_{Q}\left(r_{k}\right), U_{P}\left(r_{k}\right)=U_{Q}\left(r_{k}\right)$ and $F_{P}\left(r_{k}\right)=F_{Q}\left(r_{k}\right)$ for any $r_{k} \in R$ and $k=1,2,3, \cdots, n$. Thus $\triangle T_{k}=\triangle T_{\text {min }}=\triangle T_{\text {max }}=0$,

$\triangle C_{k}=\triangle C_{\text {min }}=\Delta C_{\max }=0, \Delta U_{k}=\Delta U_{\min }=\Delta U_{\max }=0$,

$\triangle F_{k}=\triangle F_{\text {min }}=\triangle F_{\text {max }}=0$.

Hence we get $\mathrm{K}(\mathrm{P}, \mathrm{Q})=1$.

The improved correlation coefficient formula which is defined is correct and also satisfies these properties in the above theorem . When we use any constant $\varepsilon>2$ in the following expressions

$$
\begin{aligned}
& \mu_{k}=\frac{\varepsilon-\triangle T_{k}-\triangle T_{\max }}{\varepsilon-\triangle T_{\min }-\Delta T_{\max }} \\
& \varphi_{k}=\frac{\varepsilon-\triangle C_{k}-\triangle C_{\max }}{\varepsilon-\triangle C_{\min }-\triangle C_{\max }} \\
& \delta_{k}= \frac{\varepsilon-\triangle G_{k}-\triangle G_{\max }}{\varepsilon-\triangle G_{\min }-\triangle G_{\max }} \\
& \gamma_{k}=\frac{\varepsilon-\triangle U_{k}-\triangle U_{\max }}{\varepsilon-\triangle U_{\min }-\triangle U_{\max }} \\
& v_{k}=\frac{\varepsilon-\triangle F_{k}-\triangle F_{\max }}{\varepsilon-\triangle F_{\min }-\triangle F_{\max }}
\end{aligned}
$$

Example 3.3 Let $\mathrm{A}=\{\mathrm{r}, 0,0,0,0\}$ and $\mathrm{B}=\{\mathrm{r}, 0.4,0.2,0.1,0.2\}$ be any two PNPS $\mathrm{s}$ in $R$. Therefore by equation (1) we get $K(A, B)=0.893305$. It shows that the above defined improved correlation coefficient overcome the disadvantages of the correlation coefficient .

In the following, we define a weighted correlation coefficient between PNPS s since the differences in the elements are considered into an account. Let $w_{k}$ be the weight of each element $r_{k}(k=1,2, \cdots n), w_{k} \in[0,1]$ and $\sum_{k=1}^{n} w_{k}=1$, then the weighted correlation coefficient between the PNPS's A and B 


$$
K_{w}(A, B)=\frac{1}{4} \sum_{k=1}^{n} w_{k} \mu_{k}\left(1-\triangle T_{k}\right)+\varphi_{k}\left(1-\triangle C_{k}\right)+\gamma_{k}\left(1-\triangle U_{k}\right)+v_{k}\left(1-\triangle F_{k}\right)
$$

If $w=(1 / n, 1 / n, 1 / n, \ldots 1 / n)^{T}$, then euation [4] reduces to equation [3]. $K_{w}(\mathrm{~A}, \mathrm{~B})$ also satisfies the three properties in the above theorem.

Theorem 3.4 Let $w_{k}$ be the weight for each element $r_{k}(\mathrm{k}=1,2, \cdots, \mathrm{n}), w_{k} \in[0,1]$ and $\sum_{k=1}^{n} w_{k}=1$, then the weighted correlation coefficient between the QNPS s A and $\mathrm{B}$ which is denoted by $K_{w}(\mathrm{~A}, \mathrm{~B})$ defined in equation $(\mathrm{)}$ satisfies the following properties.

1) $K_{w}(A, B)=K_{w}(B, A)$;

2) $0 \leq K_{w}(A, B) \leq 1$

3) $K_{w}(A, B)=1$ iff $\mathrm{A}=\mathrm{B}$.

It is similar to prove the properties in theorem (3.1).

\section{Decision Making using the improved correlation coefficient of QNPSs}

Multiple criteria decision making (MCDM) problems refers to make decisions when several attributes are involved in real -life problem. For example one may buy a vehicle by analysing the attributes which is given in tems of price, style,safety, comfort etc.,

Here we consider a multiple attribute decision making problem with quadripartitioned neutrosophic pythagorean information and the characteristic of an alternative $A_{i}(\mathrm{i}=1,2, \cdots m)$ on an attribute $C_{j}(\mathrm{j}=1,2, \cdots, n)$ is represented by the following PNPS s:

$$
A_{i}=\left\{C_{j}, T_{A_{i}}\left(C_{j}\right), C_{A_{i}}\left(C_{j}\right), U_{A_{i}}\left(C_{j}\right), F_{A_{i}}\left(C_{j}\right) / C_{j} \in C, j=1,2, \cdots, n\right\}
$$

Where $T_{A_{i}}\left(C_{j}\right), C_{A_{i}}\left(C_{j}\right), U_{A_{i}}\left(C_{j}\right), F_{A_{i}}\left(C_{j}\right) \in[0,1]$ and

$0 \leq T_{A_{j}}^{2}\left(C_{j}\right)+C_{A_{j}}^{2}\left(C_{j}\right)+U_{A_{j}}^{2}\left(C_{j}\right)+F_{A_{j}}^{2}\left(C_{j}\right) \leq 2$

for $C_{j} \in C, j=1,2, \cdots, n$ and $I=1,2, \cdots, m$.

To make it convenient, we are considering the following five functions $T_{A_{i}}\left(C_{j}\right), \quad C_{A_{i}}\left(C_{j}\right), \quad U_{A_{i}}\left(C_{j}\right), \quad F_{A_{i}}\left(C_{j}\right)$ in terms of pentapartitioned neutrosophic 
ISSN: 2456-8686, 5(1), 2021:142-153

https://doi.org/10.26524/cm101

pythagorean value (PNPV)

$$
d_{i j}=\left(t_{i j}, c_{i j}, u_{i j}, f_{i j}\right) \quad(i=1,2, \cdots m ; j=1,2 \cdots n)
$$

Here the values of $d_{i j}$ are usually derived from the evaluation of an alternative $A_{i}$ with respect to a criteria $C_{j}$ by the expert or decision maker. Therefore we got a pentapartitioned neutrosophic pythagorean decision matrix $D=\left(d_{i j}\right)_{m \times n}$.

In the caseof ideal alternative $A^{*}$ an ideal PNPV can be defined by

$$
d_{j}^{*}=t_{j}^{*}, c_{j}^{*}, u_{j}^{*}, f_{j}^{*}=(1,1,0,0)(j=1,2 \cdots, n) \text { in the decision making method, }
$$

Hence the weighted correlation coefficient between an alternative $A_{i}(i=1,2 \cdots, m)$ and the ideal alternative $A^{*}$ is given by,

$K_{w}\left(A_{i}, A^{*}\right)=\frac{1}{4} \sum_{j=1}^{n} w_{j}\left[\mu_{i j}\left(1-\triangle t_{i j}\right)+\varphi_{i j}\left(1-\triangle c_{i j}\right)+\gamma_{i j}\left(1-\triangle u_{i j}\right)+v_{i j}\left(1-\triangle f_{i j}\right)\right]$

Where,

$$
\begin{gathered}
\mu_{i j}=\frac{2-\triangle t_{i j}-\Delta t_{i m a x}}{2-\triangle t_{i m i n}-\triangle t_{i m a x}} \\
\varphi_{i j}=\frac{2-\triangle c_{i j}-\triangle c_{i m a x}}{2-\triangle c_{i m i n}-\triangle c_{i m a x}} \\
\gamma_{i j}=\frac{2-\triangle u_{i j}-\triangle u_{i m a x}}{2-\triangle u_{i m i n}-\triangle u_{i m a x}}, \\
v_{i j}=\frac{2-\triangle f_{i j}-\triangle f_{i m a x}}{2-\triangle f_{i m i n}-\triangle f_{i m a x}} \\
\triangle t_{i j}=\left|t_{i j}^{2}-t_{j}^{*}\right|, \\
\triangle c_{i j}=\left|c_{i j}^{2}-c_{j}^{*}\right|, \\
\triangle u_{i j}=\left|u_{i j}^{2}-u_{j}^{*}\right|, \\
\triangle t_{i m i n}=\min _{j}\left|t_{i j}^{2}-t_{j}^{*}\right|, \\
\triangle c_{i m i n}=\min _{j}\left|c_{i j}^{2}-c_{j}^{*}\right|, \\
\triangle u_{i m i n}=\min _{j}\left|u_{i j}^{2}-u_{j}^{*}\right|, \\
\triangle f_{i m i n}=\min _{j}\left|f_{i j}^{2}-f_{j}^{*}\right|,
\end{gathered}
$$


ISSN: 2456-8686, 5(1), 2021:142-153

https://doi.org/10.26524/cm101

$$
\begin{gathered}
\triangle t_{\text {imax }}=\max _{j}\left|t_{i j}^{2}-t_{j}^{*}\right|, \\
\triangle c_{\text {imax }}=\max _{j}\left|c_{i j}^{2}-c_{j}^{*}\right|, \\
\triangle u_{\text {imax }}=\max _{j}\left|u_{i j}^{2}-u_{j}^{*}\right|, \\
\triangle f_{\text {imax }}=\max _{j}\left|f_{i j}^{2}-f_{j}^{*}\right|,
\end{gathered}
$$

For $i=1,2 \cdots, m$ and $j=1,2, \cdots, n$.

By using the above weighted correlation coefficient We can derive the ranking order of all alternatives and we can choose the best one among those.

Example 4.1 This section deals the example for the multiple attribute decision making problem with the given alternatives corresponds to the criteria alloted under pentapartitioned neutrosophic pythagorean environment. For this example which we will discuss here is about the best laptop among all available alternatives based on various criteria. The alternatives $A_{1}, A_{2}, A_{3}$ respectively denotes the Lenovo, HP, Dell. The customer must take a decision according to the following four attributes that is $(1)_{1}$ is the cost (2) $C_{2}$ is the storage space $(3) C_{3}$ is the camera quality $(1) C_{4}$ is the looks. According to this attributes we will derive the ranking order of all alternatives and based on this ranking order customer will select the best one.

The weight vector of the above attributes is given by $w=(0.2,0.35,0.25,0.20)^{T}$. Here the alternatives are to be evaluated under the above four attributes by the form of QNPSs. In general the evaluation of an alternative $A_{i}$ with respect to an attribute $C_{j}(i=1,2,3 ; j=1,2,3,4)$ will be done by the questionnaire of a domain expert. In particularly, while asking the opinion about an alternative $A_{1}$ with respect to an attribute $C_{1}$, the possibility he (or) she say that the statement true is 0.3 the statement both true and false is 0.4 , the statement neither true nor false is 0.3 and the statement false is 0.4 . It can be denoted in neutrosophic notation as $d_{11}=(0.3,0.4,0.3,0.4)$. Continuing this procedure for all three alternatives with respect to four attributes we will get the following quadripartitioned single valued neutrosophic pythagorean decision value table. 
ISSN: 2456-8686, 5(1), 2021:142-153

https://doi.org/10.26524/cm101

\begin{tabular}{|c|c|c|c|c|}
\hline$A_{i} C_{j}$ & $C_{1}$ & $C_{2}$ & $C_{3}$ & $C_{4}$ \\
\hline$A_{1}$ & {$[0.3,0.4,0.3,0.4]$} & {$[0.5 .0 .1,0.2,0.1]$} & {$[0.3,0.2,0.2,0.2]$} & {$[0.4,0.5,0.3,0.2]$} \\
\hline$A_{2}$ & {$[0.4,0.3,0.2,0.1]$} & {$[0.5,0.4,0.3,0.2]$} & {$[0.6,0.2,0.3,0.2]$} & {$[0.4,0.1,0.1,0.1]$} \\
\hline$A_{3}$ & {$[0.4,0.2,0.1,0.2]$} & {$[0.3,0.3,0.2,0.1]$} & {$[0.5,0.3,0.1,0.1]$} & {$[0.1,0.4,0.3,0.2]$} \\
\hline
\end{tabular}

Then by using the proposed method we will obtain the most desirable alternative. We can get the values of the correlation coefficient $M_{w}\left(A_{i}, A^{*}\right)(i=1,2,3)$ by using Equation (3).

Hence $K_{w}\left(A_{1}, A^{*}\right)=0.51535, K_{w}\left(A_{2}, A^{*}\right)=0.53517, K_{w}\left(A_{3}, A^{*}\right)=0.5708$. Therefore the ranking order is, $A_{3}>A_{2}>A_{1}$. The alternative $A_{3}$ ( Dell) Laptop is the best choice among all the three alternatives.

\section{Conclusion}

In this paper we have defined the improved correlation coefficient of QNPSs. Decision making is a process which plays a vital role in real life problems. The main process in decision making is recognizing the problem (or) opportunity and deciding to address it. Here we have discussed the decision making method using the improved correlation coefficient of QNPSs and in particularly an illustrative example is given in multiple attribute decision making problems which involves the several alternatives based on various criteria. Hence our proposed improved correlation coefficient of QNPSs helps to identify the most suitable alternative to the customer based on the given criteria.

Funding: This research received no external funding.

Acknowledgments: I would like to express my special thanks of gratitude to S.P. Rhea and R. Kathiresan for their guidance and constant support in completing my paper.

Conflicts of Interest: The authors declare no conflict of interest.

\section{References}

[1] Atanasov K, Intuitionistic Fuzzy Sets, Fuzzy Sets and Systems, 20, 87-96 (1986).

[2] Broumi S, Smarandache F, Rough Neutrosophic sets, Ital. J. Pure. Appl. Math, 
ISSN: 2456-8686, 5(1), 2021:142-153

https://doi.org/10.26524/cm101

32, 493-502 (2014).

[3] Chiang D A and Lin N P, Correlation of fuzzy sets, Fuzzy Sets and Systems, 102, 221-226 (1999).

[4] Hong D H, Fuzzy measures for a correlation coefficient of fuzzy numbers under Tw (the weakest tnorm)-based fuzzy arithmetic operations, Information Sciences, 176, 150-160 (2006).

[5] Rajashi Chatterjee, Majumdar P and Samanta S K, On some similarity measures and entropy on quadripartitioned single valued neutrosophic sets, Journal of Intelligent and Fuzzy Systems, 30, 2475-2485 (2016).

[6] Radha R, Stanis Arul Mary A, Pentapartitioned Neutrosophic pythagorean Soft set, IRJMETS, 3, 905-914 (2021).

[7] Radha R, Stanis Arul Mary A, Pentapartitioned Neutrosophic Pythagorean Set, IRJASH, 3, 62-82 (2021).

[8] Radha R , Stanis Arul Mary A, Heptapartitioned neutrosophic sets, IRJCT, 2, 222-230 (2021).

[9] Radha R, Stanis Arul Mary A, Smarandache F, Quadripartitioned Neutrosophic Pythagorean soft set, International journal of Neutrosophic Science, 14, 09-23 (2021).

[10] Radha R , Stanis Arul Mary A, Quadripartitioned Neutrosophic Pythagorean Sets, IJRPR, 2(4), 276-281 (2021).

[11] Radha R, Stanis Arul Mary A, Neutrosophic Pythagorean soft set, Neutrosophic sets and systems, 42, 65-78 (2021).

[12] Rajashi Chatterjie, Majumdar P , Samanta S K, On similarity measures and entropy on quadripartitioned single valued neutrosophic sets,JIFS, 30, 2475-2485 (2016)..

[13] Smarandache F, A Unifying Field in Logics. Neutrosophy: Neutrosophic Probability, Set and Logic, American Research Press, Rehoboth.

[14] Wang H, Smarandache F, Zhang YQ, Sunderraman R Single valued neutrosophic sets, Multispace Multistruct , 4, 410-413 (2010). 
ISSN: 2456-8686, 5(1), 2021:142-153

https://doi.org/10.26524/cm101

[15] Wei G W, Wang H J and Lin R, Application of correlation coefficient to interval-valued intuitionistic fuzzy muo'ultiple attribute decision-making with incomplete weight information, Knowledge and Information System, 26 , 337-349 (2011).

[16] Ye J, Multicriteria fuzzy decision-making method using entropy weights-based correlation coefficients of interval valued intuitionistic fuzzy sets, Applied Mathematical Modelling, 34 , 3864-3870 (2020).

[17] Jun Ye, Another Form of Correlation Coefficient between Single Valued Neutrosophic Sets and its multiple Attribute Decision Making Method, Neutrosophic Sets and Systems, 1, 8-12. doi.org/10.5281/zenodo.571265

[18] Ye J, Multicriteria decision-making method using the correlation coefficient under single-valued neutrosophic environment, International Journal of General Systems, 2, 386-394 (2013).

[19] Ye J, Correlation coefficient of dual hesitant fuzzy sets and its application to multiple attribute decision making, Applied Mathematical Modelling, 38 , 659-666 (2013).

[20] Ye J, Improved correlation coefficients of single valued neutrosophic sets and interval neutrosophic sets for multiple attribute decision making,Journal of Intelligent and Fuzzy Systems, 27, 2453-2462 (2014).

[21] Zadeh L , Fuzzy sets, Information and Control, 8, 87-96 (1965). 